\title{
Exhibition Report
}

\section{A day at Olympia}

\section{TONY SMITH}

The curved glass roof of the exhibition hall at Olympia looks a little antiquated nowadays-and it proved far from watertight in a heavy thunderstorm during the BMA exhibition there (13-16 July). Within the hall, however, the light and space provided by the classic design showed their worth, as visitors found themselves with plenty of room to circulate in weather conditions more typical of Florida than West Kensington.

The BMA had carved out its own pale blue empire on the ground floor of the hall, the rest of which was occupied by the International Hospital and Medical Exhibition, while Exodent International displayed dental equipment and drugs upstairs. The hospital administrators, dentists, and doctors also each had their own symposia in halls in and around Olympia, so making the total enterprise awesomely complex-but giving the three disciplines a chance to eavesdrop on each other's activities.

The BMA symposium, well attended throughout the meeting despite the rail strike, ranged over a wide variety of topics from its opening session on medical information systems through deaths and disasters to nuclear war. In all over 3000 people attended the exhibition and the symposium.

\section{Aerospace medicine}

My own visit to the session of aerospace medicine proved both stimulating and informative. Firstly, Colonel Walter Cunningham, one of the astronauts in the Apollo programme, described his experiences as a guinea pig in space research and showed some fascinating film of the orbiting Skylab. Experience had shown that problems such as a demineralisation of bone, much discussed early in the space programme, had proved not to be of much practical significance. Astronauts nowadays had regular exercise periods which helped maintain muscle strength and mineral balance. "The human body adapts very well to zero gravity," said Colonel Cunningham. "The stressful time is the return to normal gravity after a period of weeks or months in space."

Zero gravity was one problem; acceleration forces were another, and Dr John Mills of the RAF Institute of Aviation Medicine described recent research into the effects on the body of forces of 4-5 $\mathrm{g}$ and higher. His dramatic film of a young pilot in a centrifuge at Farnborough firstly losing his vision and then slumping unconscious showed very clearly how susceptible the body is to rapid acceleration.

Mr Roger Green, a psychologist at the Institute of Aviation Medicine, then described some of the psychological factors underlying aircraft accidents. His pictures of the instrument displays in modern aircraft showed how complex they have become. Apparently Concorde pilots have to learn a whole series of alarm noises, indicating greater or lesser urgency, to supplement the displays of dials, counters, and warning lights. Though much modern flying consisted of passage from one radio wave to the next pilots still had to use their eyes to look for dangerand again the little time available to see and react to another aircraft was shown very clearly in a series of slides.

British Medical Journal, London WC1H 9JR

TONY SMITH, MA, BM, deputy editor

\section{Are you fit to fly?}

Dr Roger Green, the principal medical officer of British Airways, concluded the session with some practical advice to doctors asked for advice about patients' suitability for long flights. Nowadays many aircraft could stay airborne for 12 to 13 hours, said Dr Green; that was quite long enough for someone in poor health to suffer adverse effects from cerebral anoxia. Anyone

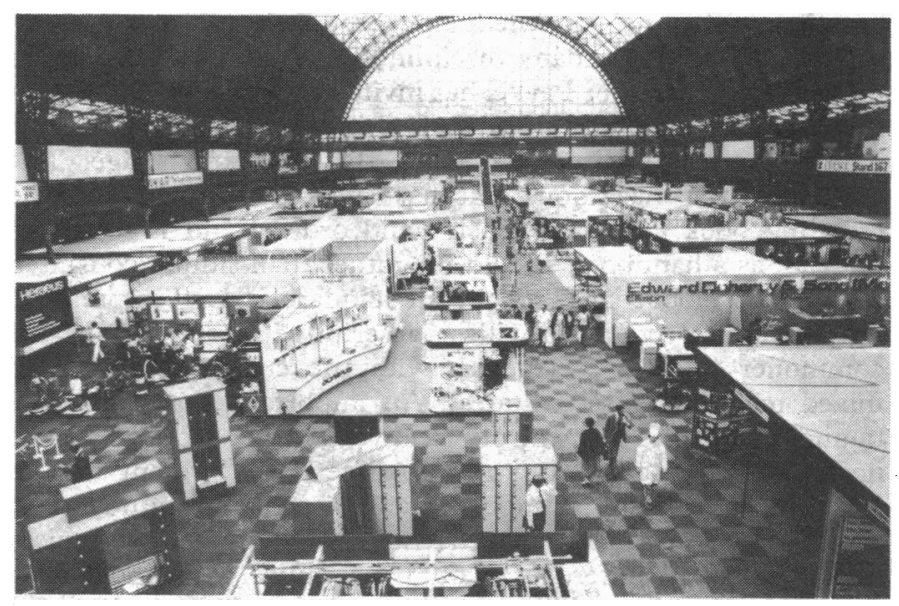

[Photograph supplied by Chris Taylor] An overall view of the exhibition hall at Olympia.

short of breath at rest or with heart failure should be assessed by an experienced clinician before embarking on a flight. Among the factors that should be taken into account were the distances that had to be travelled to and from airports at either end of the journey.

Patients recovering from surgical operations on the ear should not fly, said Dr Green; nor should women in the last month of pregnancy, or moribund patients trying to get home to die. In such cases if there were strong additional grounds for the patient needing to make the flight the airline would probably make special arrangements. Policy changed with medical advances; patients recovering from an uncomplicated myocardial infarction were now thought fit to fly within 14 days.

\section{Exhibition}

The BMA exhibition included several aspects of the Association's work and its publications-the $B M F$ and the special journals, $B M A$ Nerws, and the Family Doctor Group. These were interspersed among the stands of the pharmaceutical manufacturers, publishers, insurance companies, and instrument suppliers, providing an interesting mix of displays. Indeed the combination of the exhibition and a full day's programme at the symposium must have left many medical day trippers with little energy for a night on the town. Overall the verdict might be that the hosts had erred on the side of generosity and piled too much on the plate for the average appetite, but it was a worth while effort. 